\title{
Site-specific growth and in situ integration of different nanowire mate- rial networks on a single chip: towards a nanowire-based electronic nose for gas detection
}

\author{
Lukas Hrachowina, ${ }^{\#, \|}$ Guillem Domènech-Gil,${ }^{\S, \dagger, \|}$ Antonio Pardo, ${ }^{\S}$ Michael S. Seifner, ${ }^{\#}$ Isabel Gràcia, ${ }^{*}$ \\ Carles Cané, ${ }^{¥}$ Albert Romano-Rodríguez, ${ }^{\S, \dagger},{ }^{*}$ Sven Barth ${ }^{\#, *}$ \\ \# Institute of Materials Chemistry, TU Wien, 1060 Vienna, Austria; § Departament of Electronic and Biomedical Engi- \\ neering, Universitat de Barcelona (UB), 08028 Barcelona, Spain; ${ }^{\dagger}$ Institute of Nanoscience and Nanotechnology (IN2UB), \\ Universitat de Barcelona (UB), 08028 Barcelona, Spain; ¥ Institut de Microelectrònica de Barcelona, Centre Nacional de \\ Microelectrònica, Consejo Superior de Investigaciones Científicas (CSIC), 08193 Bellaterra, Spain.
}

KEYWORDS Nanowires; Electronic nose; Tin oxide; Tungsten Oxide; Germanium; CVD

\begin{abstract}
A new method for the site-selective synthesis of nanowires has been developed to enable material growth with defined morphology and at the same time different composition on the same chip surface. The chemical vapour deposition approach for the growth of these nanowire-based resistive devices using micromembranes can be easily modified and represents a simple, adjustable fabrication process for the direct integration of nanowire meshes in multifunctional devices. This proof-of-concept study includes the deposition of $\mathrm{SnO}_{2}, \mathrm{WO}_{3}$ and $\mathrm{Ge}$ nanowires on the same chip. The individual resistors exhibit adequate gas sensing responses towards changing gas concentration of $\mathrm{CO}, \mathrm{NO}_{2}$ and humidity diluted in synthetic air. The data have been processed by principal component analysis with cluster responses that can be easily separated and thus the devices described herein are in principle suitable for environmental monitoring.
\end{abstract}

\section{INTRODUCTION}

In this day and age there is an important societal concern about the presence of toxic and potentially harmful gases in the atmosphere, which is valid both for the industrial as well as for the outdoor and indoor environments. To carry out this monitoring, gas sensors or gas sensor systems are required, among which solid state gas sensors are an excellent choice due to their low cost and relatively high sensitivity; however, these metal oxides usually lack in selectivity due to their simultaneous response to the presence of several gases.

In general, semiconducting nanowires (NWs) have emerged as suitable components for several applications, including gas and liquid sensing, energy harvesting and generation as well as electronics and optoelectronics. ${ }^{1}$ Remarkable performances can be obtained with NW-based gas sensors in terms of sensitivity and response time. ${ }^{2}$ There are several ways for recording the actual response of a sensor device including simple optical, and electronic readout. One of the most simple and robust way to detect a sensor's response is the monitoring of the device's resistance values changing upon surface reactions with gaseous analytes, which are called chemiresistors. ${ }^{3}$ There have been tremendous improvements on device performances related to actual crystal size and additives used to facilitate the surface reactions more effectively. ${ }^{4-5}$ The high surface-to-volume ratio as well as the typically high crystallinity of NWs are beneficial for the use in chemiresistors. In this regard, the most impressive results have typically been reported for single NW-based devices, including surface decoration with inorganic particles for sensitivity enhancement, ${ }^{6}$ self-heating for ultra-low power devices, ${ }^{7}$ etc.. However, the fabrication of single NW-based devices requires time consuming contacting procedures via nanolithography ${ }^{8-10}$ or the use of dielectrophoresis to assemble NWs in specific locations ${ }^{11}$. These processes are usually quite time consuming and therefore configurations using NW networks are of particular interest. Several groups have reported the fabrication of NW-based devices using networks. ${ }^{12-13}$ Typically, these nanowires are either transferred to the sensor's surface via dry transfer, ${ }^{12}$ drop casting ${ }^{14}$ or the complete substrate is heated and the growth of NWs and other deposits covers the whole substrate ${ }^{15-16}$. As mentioned above, the remarkable performance obtained with NW devices is normally obtained on single prototypes and optimized using a trial-and-error approach. The important issue of NW-based sensor integration on multifunctional chips for their commercialization is usually not addressed and requires good control over their growth behaviour. The site-selective growth on actual sensing platforms would be a viable approach for the large scale integration of these nanostructures, but reports on NW growth without contamination of the whole growth substrate is limited to a few reports using microhotplates and micromembranes as heating elements for thermally induced growth processes. ${ }^{17-20}$

On the other hand, the well-known lack of selectivity towards gaseous species adsorbing on the surface for metal oxide based resistors as gas sensors is an issue that has to be addressed. A solution to this problem is pattern recognition across sensor arrays of different materials or temperature gradients with preferred reactions at a given temperature and surface, giving rise to a so called e-nose configuration. ${ }^{21-22}$ There are also optoelectronic e-noses or combinations of different sensor types to achieve sufficient analyte recognition. ${ }^{23} \mathrm{NW}$-based electronic noses using arrays of individual NWs have been described in literature for a single material such as $\mathrm{SnO}_{2},{ }^{24}$ surface 
modified $\mathrm{SnO}_{2} \mathrm{NWs}^{25}$ or different NW materials. ${ }^{26}$ Reproducibility, reliability, and stability of NW-based sensing devices have been investigated, however the influence on the performances of electrical contacts and their design and in situ integration during their growth has been rarely studied.

This paper demonstrates for the first time that site selective growth of different NW materials for sensing applications is possible on a single chip using chemical vapour deposition (CVD) techniques. Since all the growth stages are thermally initiated, the deposition of the NWs can be performed on a previously contaminated surfaces and the process should be compatible with complementary metal oxide semiconductor technology (CMOS) processing. This proof of concept study will pave the way for site-selective NW growth and, thus, a potential integration in actual multifunctional devices.

\section{EXPERIMENTAL SECTION}

Chemicals and Precursors. All solvents have been purchased from Sigma-Aldrich. Solvents for the synthesis of $\mathrm{Sn}\left(\mathrm{O}^{\mathrm{t}} \mathrm{Bu}\right)_{4}$ have been dried over sodium and the alkoxide precursor has been prepared as described in literature. ${ }^{27}$ Diphenylgermane (DPG) and $\mathrm{W}(\mathrm{CO})_{6}(99 \%)$ have been purchased from $\mathrm{ABCR}$ and have been used as received.

Nanowire Growth. Bulk micromachined substrates were used as a platform for the growth of NWs. The substrate chips contain 4 electrically separated $\mathrm{Si}_{3} \mathrm{~N}_{4} / \mathrm{SiO}_{2}$ micromembranes with a thickness of $1.1 \mu \mathrm{m}$ and a resistive poly-Si heater embedded within. ${ }^{28} \mathrm{Pt}$ interdigitated electrodes deposited on top of the membrane are used to electrically access the active sensing layer. The micromembranes are mounted onto a TO- 8 holder, and wire-bonded to them. The temperature has been controlled through the voltage applied to the poly-Si heater in the respective membranes. Two of the membranes have been sputtercoated with $\sim 1 \mathrm{~nm}$ Au layer to allow a seed mediated VLS-type growth, while the other two have been shadow-masked to avoid Au deposition. Ge NWs have been grown using 15 mg DPG in a low-pressure micro-CVD reactor via VLS using gold as growth seed similar to the published procedure for the growth on microhotplates at pressures of approx. $2 \times 10^{-2}$ mbar for 10 min. ${ }^{19} \mathrm{SnO}_{2} \mathrm{NWs}_{\mathrm{s}}$ have been grown on a gold coated membrane using the same procedures described in literature employing 15 $\mathrm{mg} \mathrm{Sn}\left(\mathrm{O}^{\mathrm{t}} \mathrm{Bu}\right)_{4}$ as precursor at pressures of $9 \times 10^{-2}$ mbar for 20 mins. ${ }^{17}$ The tungsten oxide NWs have been grown in home-built aerosol assisted CVD (AACVD) reactors using $15 \mathrm{ml}$ methanol as solvent for the $\mathrm{W}(\mathrm{CO})_{6}$ with a concentration of $7.58 \mathrm{mmol} / \mathrm{l}$ and carrier gas flow rates of 50-200 sccm using welding argon, while keeping the substrate temperatures at $\sim 360^{\circ} \mathrm{C}$. The temperature was determined by comparing the morphology evolution of tungsten oxides with those grown on large substrates where the substrate temperature was monitored by a thermocouple and all other parameters were held constant. For the deposition $10 \mathrm{sccm}$ argon are passed through the flask where the aerosol is generated and the rest of the argon is added to this stream above the aerosol chamber to contribute to the overall flux. This procedure was chosen to generate a sufficient vapour transport to the substrate, while controlling the precursor concentration by dilution of the generated aerosol. The deposition was carried out for $1 \mathrm{~h}$ on micromembranes without Au coating. After the growth, the complete device was three times soaked in ethanol for 2 mins to remove potential contamination due to the AACVD process. The vapour transport of the aerosol can also lead to a surface coverage with undecomposed precursor on the non-heated parts of the device. Since the precursor can easily be removed by an alcohol, this procedure was used to ensure potential remains of this carbonyl precursor. Finally, the material was heated in air for $2 \mathrm{~h}$ at $400{ }^{\circ} \mathrm{C}$ to oxidise the $\mathrm{NWs}$ to $\mathrm{WO}_{3}$, using the resistive heater for this purpose. Reference samples have been prepared in a cold-wall CVD reactor as described in literature with a modification to allow the AACVD growth. The deposition parameters have been used as benchmarks to facilitate the growth on the microhotplates.

Structural characterisation. The NWs were analysed using a FEI Inspect F50 scanning electron microscope (SEM). The Ge NWs were deposited on lacey carbon copper grids for transmission electron microscope (TEM) characterisation (Plano). In this study, a FEI TECNAI F20 operated at $200 \mathrm{kV}$ and equipped with high angle annular dark field (HAADF) STEM and EDX detector was used. The images were recorded and treated using Digital Micrograph software. X-ray diffraction (XRD) patterns have been recorded on a PANalytical XPert PRO PW 3050/60 in Bragg-Brentano geometry and $\mathrm{Cu}-\mathrm{K}_{\alpha}$ radiation. Fourier transform IR investigations were conducted on a Bruker Tensor 27 with an ATR MicroFocusing MVP-QL with a diamond crystal using OPUS version 4.0 software for analysis.

Sensor characterisation. The response of the NW containing devices towards different gases has been recorded using a home-made stainless steel chamber of $8.6 \mathrm{ml}$ volume connected to a Gometrics MGP2 gas mixer with 4 Bronkhorst Mass-Flow Controllers. Electrical measurements and flowing gas concentrations were controlled using a self-developed Labview software. For all the gas measurements a constant flow of 200 $\mathrm{ml} / \mathrm{min}$ was kept. Water vapour was added by deviating a part of the synthetic air flow through a bubbler. The air flow corresponding to each relative humidity $(\mathrm{RH})$ concentration was calibrated before testing the sensors using a commercial humidity sensor at $20^{\circ} \mathrm{C}$. Keithley 2602A dual Source Measure Units and various Keithley 2280S-32-.6 Source Measurement DC Supply units allowed to simultaneously measure the resistance of the sensors and control the voltage for heating the micromembranes for the gas sensing measurements.

The three NW-based gas sensors were characterised towards carbon monoxide $(\mathrm{CO})$, nitrogen dioxide $\left(\mathrm{NO}_{2}\right)$ and $\mathrm{RH}$ with very similar conditions of stabilisation, gas exposure and recovery times but adapting every step to the particularities of each type of sensor. The gas measurements include allowing the device 6 or 8 hours to stabilise the baseline at the operation temperature, between 0.25 and 1 hour of exposition towards the studied gases and between 0.5 and 4 hours in dry synthetic air ambient to recover the baseline after the exposition. This procedure was repeated for the different concentrations of every studied gas using one, two or three types of sensors simultaneously. The measurements were repeated at different temperatures to optimise the working temperature, except for the case of Ge NWs based sensors. Ge NWs were maintained at $100{ }^{\circ} \mathrm{C}$ to ensure a constant thickness of the $\mathrm{GeO}_{\mathrm{x}}$ layer, important to ensure a stable behaviour as gas sensor.

\section{RESULTS AND DISCUSSION}

The different NW materials have been successfully grown on micromembranes that can be individually addressed on a single chip. Each chip consists of four isolated micromembranes with low thermal mass that provides fast thermal response. Each micromembrane contains a doped polysilicon heater embedded in $\mathrm{Si}_{3} \mathrm{~N}_{4}$ membrane with an isolating layer of $\mathrm{SiO}_{2}$ encapsulating the structure and $\mathrm{Pt}$ interdigitates for electrical readout at the top surface. The details of this sensing platform are described elsewhere. ${ }^{28}$ 

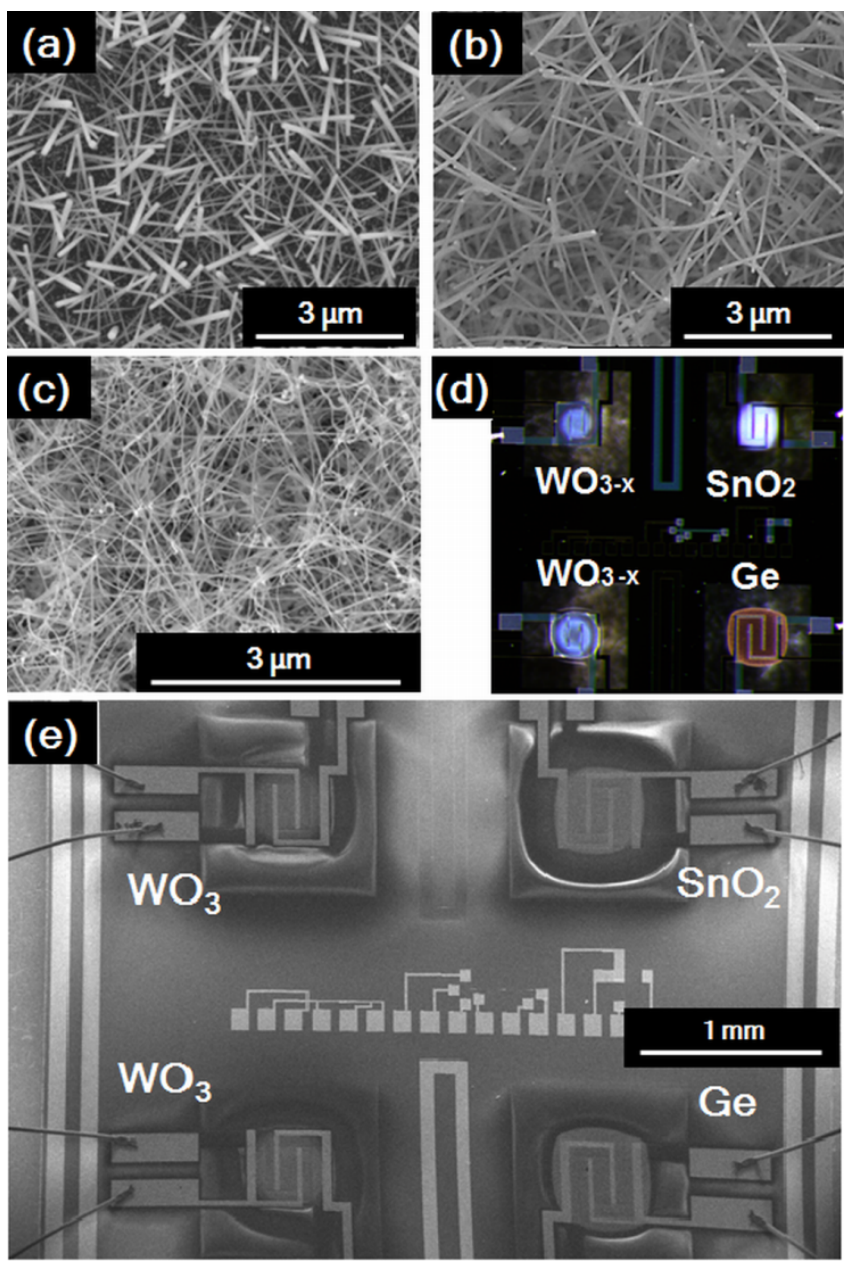

FIGURE 1: SEM images of (a) $\mathrm{W}_{18} \mathrm{O}_{49}$ NWs, (b) Ge NWs and (c) $\mathrm{SnO}_{2} \mathrm{NWs}$ site-selectively grown on micromembranes. (d) The optical image shows the blue colour of the substoichiometric tungsten oxide $\mathrm{WO}_{3-\mathrm{x}} \mathrm{NWs}$, the white $\mathrm{SnO}_{2} \mathrm{NW}$ deposit as well as a typical brown coating of Ge and (e) shows a typical SEM image with all deposits bridging the visible interdigitates on top of the membranes and the corresponding scale bar missing in the optical image. The tungsten oxide is already oxidised to $\mathrm{WO}_{3}$ in these images.

In a first step the tungsten oxide NWs are grown taking advantage of preferential nucleation of elongated substoichiometric NW-type crystals under the appropriate experimental conditions without the use of a metal growth promoter. The large number of reports on tungsten oxide NW growth for their use as sensors relies on experimental conditions that are not favourable for a site-selective growth of NWs. Typically, the processes rely on the heating of the entire device to temperatures $>400{ }^{\circ} \mathrm{C}$ in a hotwall CVD reactor, leading to "parasitic" nucleation of oxide on the entire substrate. ${ }^{29-31}$ While shadow masking can be used to channel some of the precursor flux toward preferential growth areas, it cannot fully prevent the diffusion of gaseous species and, thus, surface contamination on the devices and also the reactor walls will occur. An exception in literature depending on a local heating approach resulted in a large number of tubular structures in combination with other nanostructures and the resulting coating did not resemble the products obtained in hotwall reactor CVD, where the whole device was exposed to heat. ${ }^{32} \mathrm{We}$ achieved site-selective growth of tungsten oxide NWs exclusively in the heated area of a micromembrane by aerosol assisted CVD using a methanol solution of tungsten hexacarbonyl. The best results were obtained when the solution was stirred for one hour prior to deposition, which leads to a partial CO ligand exchange to coordinated methanol triggered by UV light. ${ }^{33}$ The SEM image in Fig. 1a clearly illustrates the formation of tungsten oxide NWs in the process and shows that these NWs can be grown on site-selectively heated areas in a cold-wall reactor. Fine-tuning of the deposition temperature allows the exclusive formation of NWs, without a particle co-deposit on the membranes and NWs (Fig. S1). The sub-stoichiometric $\mathrm{WO}_{3-\mathrm{x}} \mathrm{NWs}$ are highly crystalline and display a well-known growth direction along the $\langle 010\rangle$ axis in TEM images and the corresponding fast Fourier transformations (FFT) that are consistent with monoclinic $\mathrm{W}_{18} \mathrm{O}_{49}$ (Fig. S2). A high number of stacking faults perpendicular to the growth axis are a well-known phenomenon in tungsten oxide and tungsten suboxide NWs. ${ }^{34-35}$ The presence of stacking faults is visible in the FFT pattern showing a streaking of the spots perpendicular to the growth direction (Fig. S2). The initially blue NW deposits can be oxidised by a short heat treatment for $2 \mathrm{~h}$ at $400{ }^{\circ} \mathrm{C}$ in air leading to visually white deposits. The overall morphology does not change by this oxidation procedure, while the conversion can be followed by XRD showing a shift in the dominating reflection towards lower $2 \theta$ values (Fig. S3). The high crystallinity of the tungsten oxide NWs remains, as shown in XRD, and is also illustrated by HRTEM images of single NWs and their corresponding FFT images (Fig. $\mathrm{S} 2 \mathrm{~b})$.

Growth procedures of $\mathrm{Ge}$ and $\mathrm{SnO}_{2} \mathrm{NWs}$ on micromembranes and microhotplates have been previously described. ${ }^{17,} 19$ Ge NW growth has been achieved on micromembranes, as shown in the SEM image in Fig. 1b, using Au as growth seed and DPG as precursor and keeping the substrate at $\sim 400{ }^{\circ} \mathrm{C} .{ }^{19}$ Lower temperatures lead to curly and defect rich NWs, while temperatures, which are too high result in a larger number of NWs growing in plane of the substrate. Both regimes have been mapped out and have been avoided, because especially the surface migrating NWs will lead to conduction paths with small surface area and, therefore, are less suitable for sensor operation. The as-grown Ge NWs are single crystalline, as shown in Fig. S4a. The DPG precursor does not react with hydroxyl groups on the surface and, therefore, contamination issues can be neglected, even when tungsten oxide NWs have been prior deposited on a different membrane of the same chip. Finally, tin oxide NWs have been grown on the remaining micromembrane using $\mathrm{Au}$ as growth promoter and $\mathrm{Sn}\left(\mathrm{O}^{t} \mathrm{Bu}\right)_{4}$ as precursor. The HRTEM image in Figure S4b shows the single crystalline nature of the $\mathrm{SnO}_{2} \mathrm{NWs} .{ }^{17}$ In a previous study, we mentioned the absence of a surface bound $\mathrm{SnO}_{2}$ particle layer for $\mathrm{SnO}_{2} \mathrm{NW}$ growth, ${ }^{18}$ but further investigation is required to understand the low tendency of thin film formation even though in this temperature regime non-catalysed precursor decomposition occurs. ${ }^{36}$ An expected $\mathrm{SnO}_{2}$ particle film forms at lower temperatures by heating a membrane without the gold growth seed (Fig. S5). Increasing the deposition temperature reveals a clean silicon oxide surface of the membrane in the centre, which is generally slightly higher in temperature than the outer heated part of the membrane that remains with a $\mathrm{SnO}_{2}$ particle layer. At the $\mathrm{SnO}_{2}$ NW growth temperatures the whole inner part is so hot that desorption dominates under the given experimental conditions including a low precursor concentration. Therefore, this absence of a particle film can be attributed to a thermal desorption process at elevated temperatures, which is in accordance with the generally accepted dependence of growth rates on the temperature for a given precursor flux. ${ }^{37}$ The Au-supported NW nucleation leads to a quick formation of single crystalline material, which will not easily desorb and the NW product remains on 
the membrane. Fig. 1d and 1e show optical and SEM images of one chip with the 4 membranes on top of which the different NWs have been grown. Cross section SEM images in Fig. S6 show the length of the NWs grown on the micromembranes, which can be used to for the calculation of the surface area being in the range of $\sim 5-20 \mathrm{~m}^{2} / \mathrm{g}\left(\mathrm{WO}_{3} \sim 5.6 \mathrm{~m}^{2} / \mathrm{g} ; \mathrm{Ge} \sim 9.4 \mathrm{~m}^{2} / \mathrm{g}\right.$; $\mathrm{SnO}_{2} \sim 19.5 \mathrm{~m}^{2} / \mathrm{g}$ ).
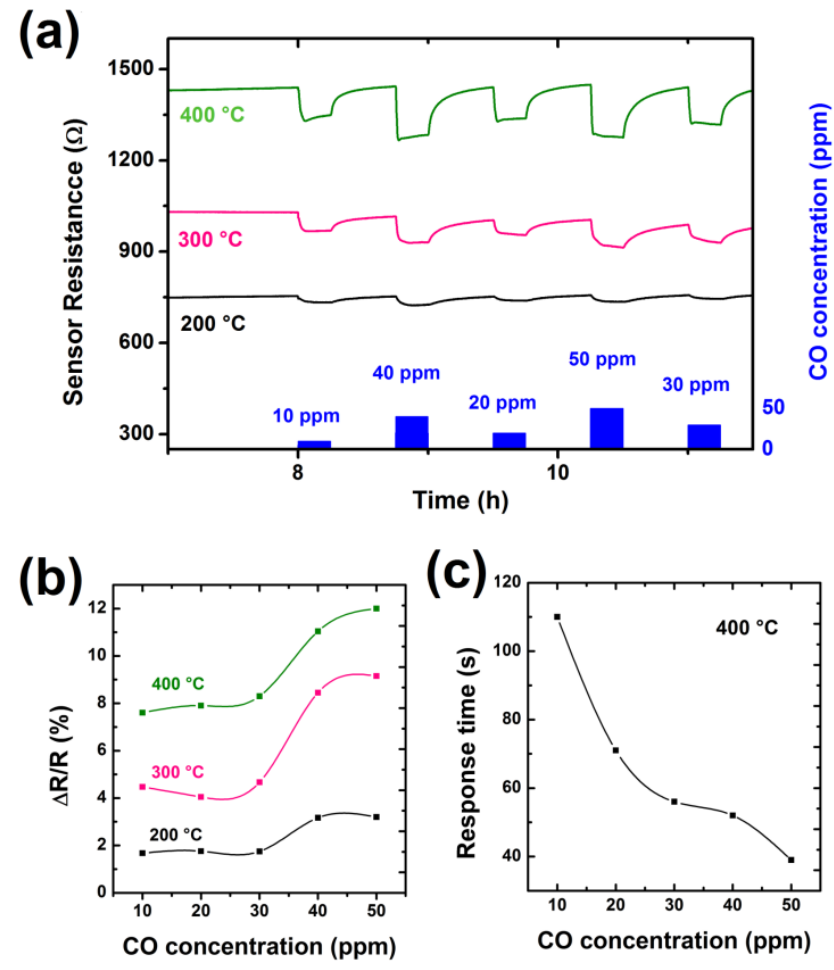

FIGURE 2: (a) Evolution of $\mathrm{SnO}_{2} \mathrm{NW}$ device resistance for different concentrations of $\mathrm{CO}$ in synthetic air. The response of the results from (a) are shown in (b) as function of concentration at different temperatures and in (c) the $\mathrm{CO}$ concentration dependence of response time at 400 .

The response of the sensors towards the different adsorbing species can be defined as the relative change in the sensor's resistance, which is expressed in general terms as illustrated in equation 1 , irrespective from oxidizing or reducing gases or $\mathrm{p}$ or n-type semiconductors:

$$
\operatorname{Response}(\%)=\frac{\left|R_{\text {air }}-R_{\text {gas }}\right|}{R_{\text {air }}}
$$

The sensor's response time is defined as the time required to reach $90 \%$ of the steady state resistance under a given gaseous concentration, while the recovery time is the time between the gas flow stops and the resistance varies $90 \%$ of the interval between the steady state and the base resistance. The sensors response towards the different gas species was tested either individually or with two or three sensors working simultaneously. Comparisons between individual and simultaneous measurements reveal no differences, proving that the proximity between operating sensors has no influence on the actual readout of the individual devices. $\mathrm{SnO}_{2}$ and $\mathrm{WO}_{3}$ are typically n-type semiconductors and, therefore, surface reactions with the same gas will show similar trends in respect to resistance changes. ${ }^{38-43}$ The response of Ge NWs is typically reversed when compared to the aforementioned oxides due to the fact that this material is usually a p-type semiconductor. ${ }^{19}$ All the measurements in this study are carried out in synthetic air because the absence of oxygen alters the sensors' response dramatically and base resistance values are highly dependent on the chemisorption of oxygen at their surface, resulting in an altered space charge region at the surface. ${ }^{44}$

The change in resistance of the locally grown $\mathrm{SnO}_{2} \mathrm{NWs}$ meshes against varying $\mathrm{CO}$ concentration in synthetic air was investigated in the range of 10 to $50 \mathrm{ppm}$, which is very close to the legal limit for $8 \mathrm{~h}$ exposure time-weighted average (TWA) of $8.6 \mathrm{ppm} \mathrm{CO} .{ }^{45}$ The evolution of the $\mathrm{SnO}_{2}$ sensor's resistance at different temperatures is represented in Fig. 2a. The decrease in resistance upon $\mathrm{CO}$ exposure is expected for an n-type semiconductor gas sensor such as $\mathrm{SnO}_{2}$. The shift in baseline can be attributed to different chemisorbed oxygen species on the $\mathrm{SnO}_{2}$ surface. In the temperature range $200-400^{\circ}$ the dissociation of initially molecular $\left(\mathrm{O}_{2}^{-}\right)$to atomic $\left(\mathrm{O}^{-}\right)$chemisorbed oxygen species leads to an increased number of charges captured from the semiconductor and, thus, an increase in the resistance. ${ }^{19,46-}$ ${ }^{47}$ Response of the $\mathrm{SnO}_{2} \mathrm{NW}$ device towards $\mathrm{CO}$ up to $50 \mathrm{ppm}$ in concentration as a function of temperature is illustrated in Fig. 2b. The site-selectively grown $\mathrm{SnO}_{2} \mathrm{NW}$ sensors show a response of up to $12 \%$ for $50 \mathrm{ppm}$ of $\mathrm{CO}$ at $400{ }^{\circ} \mathrm{C}$, accompanied by a response time as low as 40 seconds. At these temperatures the sensor's response is at a maximum and also the response time is lowest. A plot of the response time as a function of concentration is represented in Fig. 2c and its decrease with increasing $\mathrm{CO}$ concentration is observed. Non-linear responses are well known for semiconductor gas sensors and can be typically represented by a power law. ${ }^{48}$ The response of this sensor towards $\mathrm{NO}_{2}$ shows the well-known increase in resistance upon exposure, typical of an n-type semiconductor towards an oxidizing atmosphere in dry synthetic air at $300{ }^{\circ} \mathrm{C}$ (Fig. S7). This range is slightly higher than the upper time-weighted average exposure limit in the EU $(0.1 \mathrm{ppm})$ for air quality standards in urban areas and one day exposure times ${ }^{49}$; however, in specific areas such as underground parking garages ${ }^{50}$ or ice arenas ${ }^{51}$ the values can be higher. However, responses of $56 \%$ towards 0.2 ppm $\mathrm{NO}_{2}$, as illustrated in Fig. S7b, demonstrate that these values can be easily detected with the here presented devices. Fig. $\mathrm{S} 8$ shows the response of the $\mathrm{SnO}_{2} \mathrm{NW}$ meshes towards relative humidity. The interaction with the oxide surface leads to a reduction of resistance in the $\mathrm{SnO}_{2} \mathrm{NW}$-based devices which is in agreement with reports on single NW resistors ${ }^{52}$ as well as NW meshes ${ }^{16}$.

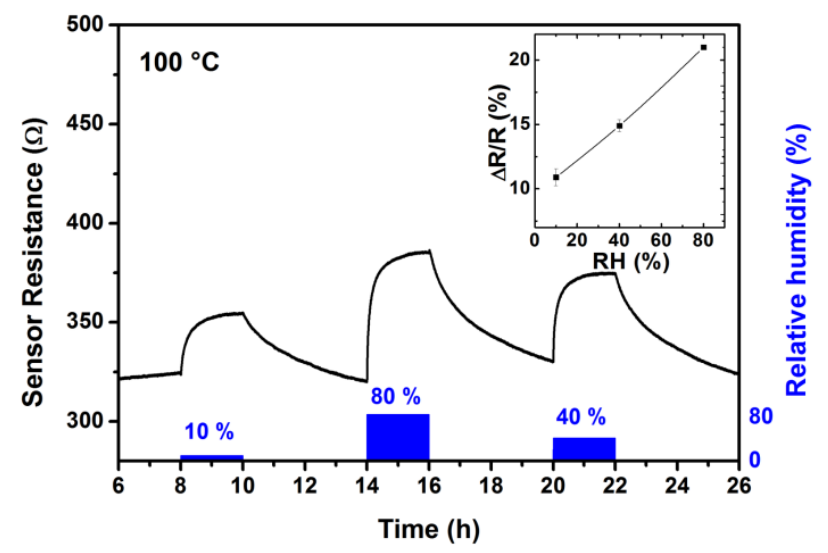

FIGURE 3: Response of Ge NWs towards changes in the humidity levels in synthetic air at $100{ }^{\circ} \mathrm{C}$. The resistance changes are linear as shown in the inset. 
We recently demonstrated that Ge NW-based resistors based on micro-hotplates can be successfully used as humidity sensors. ${ }^{19}$ The Ge NW containing micromembranes, as shown in Fig. 1, have been used as humidity sensors revealing a linear response towards different humidity levels (Fig. 3). The dynamic response is rather slow but reliable and reproducible. A noticeable difference to our previous study on microhotplates is the increased cross-sensitivity against other gases such as $\mathrm{CO}$ (response $0.8 \%$ for $50 \mathrm{ppm} \mathrm{CO}$; previously $0.8 \%$ for $150 \mathrm{ppm}$ CO) and $\mathrm{NO}_{2}$ (11\% for 1 ppm $\mathrm{NO}_{2}$, which was merely $1.7 \%$ observed before), as shown in Figs. S9 and S10. This is most likely due to a slight surface modification of the Ge NWs by $\mathrm{SnO}_{2}$ residues that were deposited due to the previously mentioned thermally activated desorption of $\mathrm{SnO}_{2}$ during the growth. According to these results and in order to optimise the NW-based devices, the fabrication process could be changed slightly to avoid the obvious modification of the material on the surface. In future experiments a short plasma treatment will be applied after the tin oxide deposition to remove any unwanted contamination.

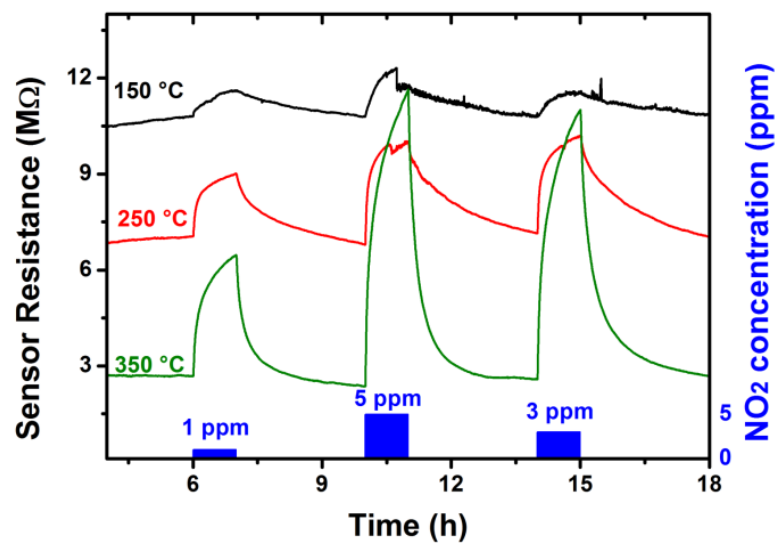

FIGURE 4: Response of $\mathrm{WO}_{3} \mathrm{NWs}$ towards different concentrations of $\mathrm{NO}_{2}$ at temperatures of $150{ }^{\circ} \mathrm{C}, 250^{\circ} \mathrm{C}$ and $350{ }^{\circ} \mathrm{C}$.

Tungsten oxides are well known for their electrical response towards different gaseous species. ${ }^{53-54}$ The response of tungsten oxide NW meshes towards $\mathrm{NO}_{2}$ is highly temperature dependent, as demonstrated in Fig. 4. The measured responses are in the range of more than $300 \%$ for $\mathrm{NO}_{2}$ concentrations of $\geq$ $3 \mathrm{ppm}$ in synthetic air at a temperature of $350^{\circ} \mathrm{C}$. However, the response towards $\mathrm{CO}$ is moderate $(<10 \%)$ as illustrated in Fig. S11. The reduction of resistance in $\mathrm{WO}_{3} \mathrm{NW}$ devices during the interaction with water molecules is up to $69 \%$ for a relative humidity of $70 \%$, keeping the sensor at $250{ }^{\circ} \mathrm{C}$ (Fig. S12).

However, at real ambient conditions the sensors are not exposed to single gases but to complex gas mixtures, where it is difficult to deduce the contribution of the individual species. This scenario is illustrated in Fig. 5a, showing the response of a $\mathrm{NW}$-based $\mathrm{SnO}_{2}$ gas sensor towards mixtures of $\mathrm{CO}, \mathrm{NO}_{2}$ and humidity in the same concentrations as described before. It is clear that the responses obtained are not simply the sum of the response to the individual gases, but that these gases can compete for the same adsorption sites and, consequently, react differently as for one single gas. This makes the sensing of gas mixtures using metal oxides quite complicated and electronic noses are required for their discrimination. (a)

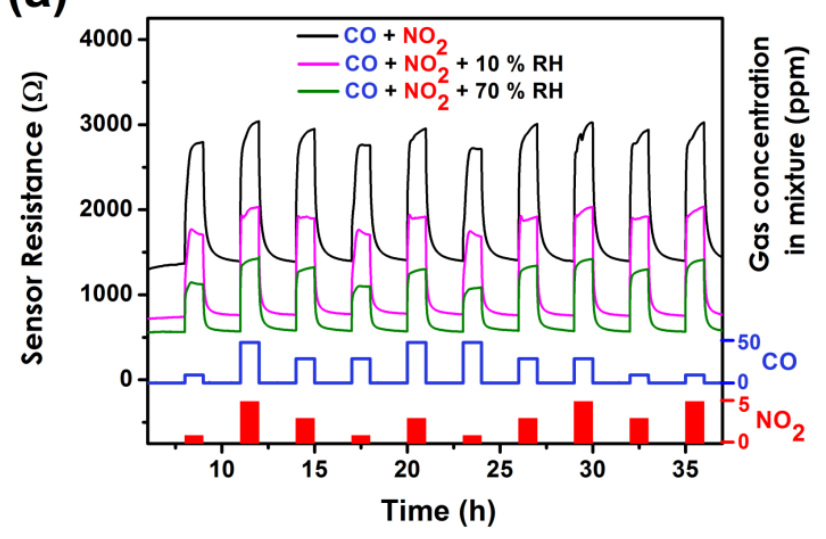

(b)

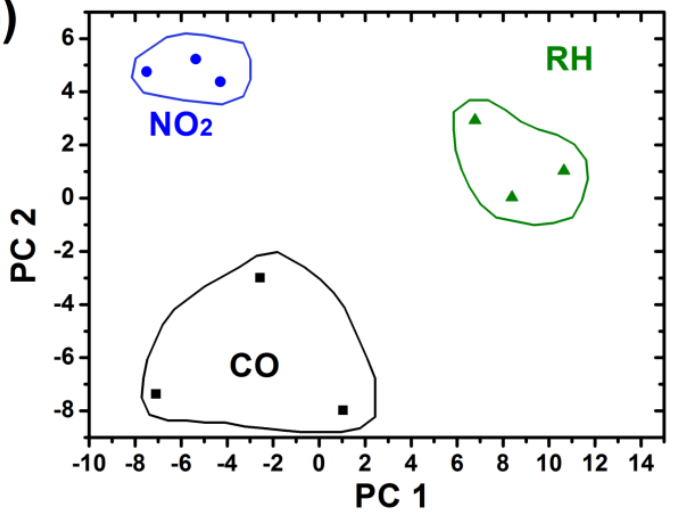

FIGURE 5: (a) Resistance of a $\mathrm{SnO}_{2} \mathrm{NW}$-based device in changing environment of gas mixtures composed of $\mathrm{CO}, \mathrm{NO}_{2}$ and relative humidity with variable concentrations in synthetic air at $250^{\circ} \mathrm{C}$. (b) Principal component analysis for $\mathrm{SnO}_{2}, \mathrm{WO}_{3}$ and $\mathrm{Ge}$ NW-based gas sensor towards carbon monoxide, nitrogen dioxide and relative humidity clearly showing the different clustering of the measured data. The actual area illustrated is for included guidance of the eyes only.

For this reason it is necessary that the observed response of the chemiresistors is monitored using more complex analysis to determine the concentrations of different analytes in gas mixtures. Principal component analysis (PCA) is mathematically defined as an orthogonal linear transformation. This transformation changes the data to a new coordinate system such that the greatest variances are projected to lie in the first and second axes. ${ }^{55-56}$ The PCA method helps classifying different gas species using clustering, and helps, visually, to easily identify the ability of the sensor array to distinguish between the analyzed gases. The main objective of this type of work is to determine the cluster in which a studied gas resides and to quantify its presence. In our principal component analysis (PCA) study, the results from $\mathrm{SnO}_{2}, \mathrm{WO}_{3}$ and $\mathrm{Ge} \mathrm{NW}$-based gas sensors, working at 300,250 and $100{ }^{\circ} \mathrm{C}$ respectively, towards CO (10 to 50 ppm), $\mathrm{NO}_{2}$ (1 to $5 \mathrm{ppm}$ ) and $\mathrm{RH}$ (from 0 to $80 \%$ ) reveal 3 different clusters corresponding to each gas species, as shown in Fig. 5b. The existence of different clusters and their obvious separation in the PCA representation proves the capacity of the three-sensor system to distinguish between these three gases in the measured concentration range, which can be harmful for human health. The next generation of such NW devices is expected to be even more efficient in discriminating between different gases, when the processes will be further optimised and potentially a fourth NW material incorporated. The NW-based 
devices do also respond to gases such as ethanol or ammonia as shown in Fig. S13 and Fig. S14 and thus their field of application can be much broader. A detailed study of the gas sensor response, the PCA analysis in the presence of several gases in the atmosphere and the corresponding discrimination properties of this system is under preparation and will be published elsewhere.

\section{CONCLUSION}

The here presented proof-of-concept study related to the in situ preparation of a NW-based sensor system by different CVD approaches shows clearly that the methodology can be successfully applied. Three different materials are demonstrated to be grown on one single chip in a defined geometry with little interference on the growth parameters caused by the prior deposition of other NW material. The use of CVD techniques are compatible with CMOS processing and, therefore, the impact of preparing gas sensors on any kind of chips for multifunctional devices is intriguing. The sensors herein show responses similar to those of individual devices prepared exclusively on single micromembranes when exposed to atmospheres containing known harmful gases, such as $\mathrm{CO}$ and $\mathrm{NO}_{2}$, and at different levels of relative humidity. Some improvements to the sensors can be already imagined including an additional site-selective surface decoration with metal or metal oxide particles for a well-known sensitivity improvement ${ }^{5,30}$ by thermal CVD using appropriate precursors. However, even at this stage the discrimination between all the three analytes in the environment is obtained by the well-known PCA representation and the cluster responses can be easily distinguished.

\section{ASSOCIATED CONTENT}

Supporting Information. Supporting TEM, SEM and XRD images are provided to illustrate the quality of the NWs prepared by CVD and used in this study as gas sensor material. Moreover, additional information on gas responses of the different materials described herein is provided.

\section{AUTHOR INFORMATION}

\section{Corresponding Author}

* Email: S. B.: sven.barth@tuwien.ac.at.

A.R.R.: aromano@el.ub.edu

" L.H. and G. D.-G. contributed equally to this paper.

\section{ACKNOWLEDGMENT}

We would like to thank the University Service Center for TEM (USTEM) for access to the electron microscopes at TU Wien. Financial support of TU Wien is gratefully acknowledged. This work has been partially supported by the Spanish Ministerio de Economía y Competitividad, through projects TEC2013-48147-C6 and TEC2016-79898-C6 (AEI/FEDER, EU).

\section{REFERENCES}

1. Barth, S.; Hernandez-Ramirez, F.; Holmes, J. D.; RomanoRodriguez, A., Synthesis and Applications of One-Dimensional Semiconductors. Prog. Mater. Sci. 2010, 55, 563-627.

2. Comini, E.; Baratto, C.; Faglia, G.; Ferroni, M.; Vomiero, A.; Sberveglieri, G., Quasi-One Dimensional Metal Oxide Semiconductors: Preparation, Characterization and Application as Chemical Sensors. Prog. Mater. Sci. 2009, 54, 1-67.
3. Seiyama, T.; Kato, A.; Fujiishi, K.; Nagatani, M., A New Detector for Gaseous Components Using Semiconductive Thin Films. Anal. Chem. 1962, 34, 1502-1503.

4. Yamazoe, N., New Approaches for Improving Semiconductor Gas Sensors. Sens. Actuators B 1991, 5, 7-19.

5. Degler, D.; Rank, S.; Müller, S.; Pereira de Carvalho, H. W.; Grunwaldt, J.-D.; Weimar, U.; Barsan, N., Gold-Loaded Tin Dioxide Gas Sensing Materials: Mechanistic Insights and the Role of Gold Dispersion. ACS Sensors 2016, 1, 1322-1329.

6. Kolmakov, A.; Klenov, D. O.; Lilach, Y.; Stemmer, S.; Moskovits, M., Enhanced Gas Sensing by Individual SnO2 Nanowires and Nanobelts Functionalized with Pd Catalyst Particles. Nano Letters 2005, 5, 667-673.

7. Prades, J. D.; Jimenez-Diaz, R.; Hernandez-Ramirez, F.; Barth, S.; Cirera, A.; Romano-Rodriguez, A.; Mathur, S.; Morante, J. R., Ultralow Power Consumption Gas Sensors based on Self-Heated Individual Nanowires. Appl. Phys. Lett. 2008, 93, 123110.

8. Kolmakov, A.; Zhang, Y.; Cheng, G.; Moskovits, M., Detection of $\mathrm{CO}$ and $\mathrm{O} 2$ Using Tin Oxide Nanowire Sensors. $A d v$. Mater. 2003, 15, 997-1000.

9. Hernández-Ramírez, F.; Tarancón, A.; Casals, O.; Rodríguez, J.; Romano-Rodríguez, A.; Morante, J. R.; Barth, S.; Mathur, S.; Choi, T. Y.; Poulikakos, D.; Callegari, V.; Nellen, P. M., Fabrication and Electrical Characterization of Circuits Based on Individual Tin Oxide Nanowires. Nanotechnology 2006, 17, 5577.

10. Domènech-Gil, G.; Barth, S.; Samà, J.; Pellegrino, P.; Gràcia, I.; Cané, C.; Romano-Rodriguez, A., Gas sensors based on individual indium oxide nanowire. Sens. Actuators B 2017, 238, 447 454.

11. Freer, E. M.; Grachev, O.; Duan, X.; Martin, S.; Stumbo, D. P., High-Yield Self-Limiting Single-Nanowire Assembly with Dielectrophoresis. Nat. Nanotechnol. 2010, 5, 525-530.

12. Sysoev, V. V.; Schneider, T.; Goschnick, J.; Kiselev, I.; Habicht, W.; Hahn, H.; Strelcov, E.; Kolmakov, A., Percolating SnO2 Nanowire Network as a Stable Gas Sensor: Direct Comparison of Long-Term Performance Versus SnO2 Nanoparticle Films. Sens. Actuators B 2009, 139, 699-703.

13. Ponzoni, A.; Comini, E.; Sberveglieri, G.; Zhou, J.; Deng, S. Z.; Xu, N. S.; Ding, Y.; Wang, Z. L., Ultrasensitive and Highly Selective Gas Sensors using Three-Dimensional Tungsten Oxide Nanowire Networks. Appl. Phys. Lett. 2006, 88, 203101.

14. Chávez, F.; Pérez-Sánchez, G. F.; Goiz, O.; Zaca-Morán, P.; Peña-Sierra, R.; Morales-Acevedo, A.; Felipe, C.; Soledad-Priego, M., Sensing Performance of Palladium-Functionalized WO3 Nanowires by a Drop-Casting Method. Appl. Surf. Sci. 2013, 275, 28-35.

15. Vomiero, A.; Ponzoni, A.; Comini, E.; Ferroni, M.; Faglia, G.; Sberveglieri, G., Direct Integration of Metal Oxide Nanowires into an Effective Gas Sensing Device. Nanotechnology 2010, 21, 145502.

16. Choi, Y.-J.; Hwang, I.-S.; Park, J.-G.; Choi, K. J.; Park, J.H.; Lee, J.-H., Novel Fabrication of an $\mathrm{SnO} 2$ Nanowire Gas Sensor with High Sensitivity. Nanotechnology 2008, 19, 095508.

17. Barth, S.; Jimenez-Diaz, R.; Sama, J.; Daniel Prades, J.; Gracia, I.; Santander, J.; Cane, C.; Romano-Rodriguez, A., Localized Growth and in situ Integration of Nanowires for Device Applications. Chem. Commun. 2012, 48, 4734-4736.

18. Samà, J.; Barth, S.; Domènech-Gil, G.; Prades, J.-D.; López, N.; Casals, O.; Gràcia, I.; Cané, C.; Romano-Rodríguez, A., SiteSelectively Grown SnO2 NWs Networks on Micromembranes for Efficient Ammonia Sensing in Humid Conditions. Sens. Actuators $B$ 2016, 232, 402-409.

19. Samà, J.; Seifner, M. S.; Domènech-Gil, G.; Santander, J.; Calaza, C.; Moreno, M.; Gràcia, I.; Barth, S.; Romano-Rodríguez, A., Low Temperature Humidity Sensor Based on Ge Nanowires Selectively Grown on Suspended Microhotplates. Sens. Actuators B 2017, 243, 669-677.

20. Steinhauer, S.; Brunet, E.; Maier, T.; Mutinati, G. C.; Köck, A. In On-Chip Synthesis of CuO Nanowires for Direct Gas Sensor Integration, 2012 12th IEEE International Conference on Nanotechnology (IEEE-NANO), 20-23 Aug. 2012; 2012; pp 1-4.

21. Persaud, K.; Dodd, G., Analysis of Discrimination Mechanisms in the Mammalian Olfactory System using a Model Nose. Nature 1982, 299, 352. 
22. Wilson, A.; Baietto, M., Applications and Advances in Electronic-Nose Technologies. Sensors 2009, 9, 5099.

23. Li, Z.; Suslick, K. S., Portable Optoelectronic Nose for Monitoring Meat Freshness. ACS Sensors 2016, 1, 1330-1335.

24. Sysoev, V. V.; Goschnick, J.; Schneider, T.; Strelcov, E.; Kolmakov, A., A Gradient Microarray Electronic Nose Based on Percolating SnO2 Nanowire Sensing Elements. Nano Lett. 2007, 7, 3182-3188.

25. Baik, J. M.; Zielke, M.; Kim, M. H.; Turner, K. L.; Wodtke, A. M.; Moskovits, M., Tin-Oxide-Nanowire-Based Electronic Nose Using Heterogeneous Catalysis as a Functionalization Strategy. ACS Nano 2010, 4, 3117-3122.

26. Sysoev, V. V.; Button, B. K.; Wepsiec, K.; Dmitriev, S.; Kolmakov, A., Toward the Nanoscopic "Electronic Nose": Hydrogen vs Carbon Monoxide Discrimination with an Array of Individual Metal Oxide Nano- and Mesowire Sensors. Nano Letters 2006, 6, 1584-1588.

27. Hampden-Smith, M. J.; Wark, T. A.; Rheingold, A.; Huffman, J. C., Solid State and Solution Structural Investigation of Homoleptic Tin(IV) Alkoxide Compounds. Part I. Sn(O-t-Bu)4 and [Sn(O-i-Pr)4•HO-i-Pr]2. Can. J. Chem. 1991, 69, 121-129.

28. Puigcorbé, J.; Vogel, D.; Michel, B.; Vilà, A.; Gràcia, I.; Cané, C.; Morante, J. R., Thermal and Mechanical Analysis of Micromachined Gas Sensors. J. Micromech. Microeng. 2003, 13, 548.

29. Molkenova, A.; Sarip, R.; Sathasivam, S.; Umek, P.; Vallejos, S.; Blackman, C.; Hogarth, G.; Sankar, G., Single-Step CoDeposition of Nanostructured Tungsten Oxide Supported Gold Nanoparticles using a Gold-Phosphine Cluster Complex as the Gold Precursor. Sci. Technol. Adv. Mater. 2014, 15, 065004.

30. Vallejos, S.; Umek, P.; Stoycheva, T.; Annanouch, F.; Llobet, E.; Correig, X.; De Marco, P.; Bittencourt, C.; Blackman, C., Single-Step Deposition of Au- and Pt-Nanoparticle-Functionalized Tungsten Oxide Nanoneedles Synthesized Via Aerosol-Assisted CVD, and Used for Fabrication of Selective Gas Microsensor Arrays. Adv. Funct. Mater. 2013, 23, 1313-1322.

31. Stoycheva, T.; Annanouch, F. E.; Gràcia, I.; Llobet, E.; Blackman, C.; Correig, X.; Vallejos, S., Micromachined Gas Sensors Based on Tungsten Oxide Nanoneedles Directly Integrated Via Aerosol Assisted CVD. Sens. Actuators B 2014, 198, 210-218.

32. Annanouch, F. E.; Gràcia, I.; Figueras, E.; Llobet, E.; Cané, C.; Vallejos, S., Localized Aerosol-Assisted CVD of Nanomaterials for the Fabrication of Monolithic Gas Sensor Microarrays. Sens. Actuators $B$ 2015, 216, 374-383.

33. Zhu, L.; Saha, S.; Wang, Y.; Keszler, D. A.; Fang, C., Monitoring Photochemical Reaction Pathways of Tungsten Hexacarbonyl in Solution from Femtoseconds to Minutes. J. Phys. Chem. B 2016, 120, 13161-13168.

34. Klinke, C.; Hannon, J. B.; Gignac, L.; Reuter, K.; Avouris, P., Tungsten Oxide Nanowire Growth by Chemically Induced Strain. J. Phys. Chem. B 2005, 109, 17787-17790.

35. Rao, P. M.; Zheng, X., Flame Synthesis of Tungsten Oxide Nanostructures on Diverse Substrates. Proc. Combust. Inst. 2011, 33, 1891-1898.

36. Mathur, S.; Barth, S., Molecule-Based Chemical Vapor Growth of Aligned SnO2 Nanowires and Branched SnO2/V2O5 Heterostructures. Small 2007, 3, 2070-2075.

37. Choy, K. L., Chemical Vapor Deposition of Coatings. Prog. Mater. Sci. 2003, 48, 57-170.

38. Gaggiotti, G.; Galdikas, A.; Kačiulis, S.; Mattogno, G.; Šetkus, A., Surface Chemistry of Tin Oxide Based Gas Sensors. J. Appl. Phys. 1994, 76, 4467-4471.

39. Weimar, U.; Göpel, W., A.C. Measurements on Tin Oxide Sensors to Improve Selectivities and Sensitivities. Sens. Actuators B 1995, 26, 13-18.
40. Polleux, J.; Gurlo, A.; Barsan, N.; Weimar, U.; Antonietti, M.; Niederberger, M., Template-Free Synthesis and Assembly of Single-Crystalline Tungsten Oxide Nanowires and their Gas-Sensing Properties. Angew. Chem. 2006, 118, 267-271.

41. Vallejos, S.; Di Maggio, F.; Shujah, T.; Blackman, C., Chemical Vapour Deposition of Gas Sensitive Metal Oxides. Chemosensors 2016, 4, 4.

42. Bârsan, N.; Weimar, U., Understanding the Fundamental Principles of Metal Oxide Based Gas Sensors; the Example of CO Sensing with SnO2 Sensors in the Presence of Humidity. J. Phys.: Condens. Matter 2003, 15, R813.

43. Zou, Y.; Chen, S.; Sun, J.; Liu, J.; Che, Y.; Liu, X.; Zhang, J.; Yang, D., Highly Efficient Gas Sensor Using a Hollow SnO2 Microfiber for Triethylamine Detection. ACS Sensors 2017, 2, 897 902.

44. Yamazoe, N.; Sakai, G.; Shimanoe, K., Oxide Semiconductor Gas Sensors. Catal. Surv. Asia 2003, 7, 63-75.

45. Danzon, M. A.; Leeuwen, R. V.; Krzyzanowski, M., Air Quality Guidelines for Europe. Second Edition ed.; World Health Organization: Copenhagen, 2000.

46. Ahlers, S.; Becker, T.; Hellmich, W.; Braunmühl, C. B.-v.; Müller, G., Temperature- and Field-Effect-Modulation Techniques for Thin-Film Metal Oxide Gas Sensors. In Advanced Gas Sensing: The Electroadsorptive Effect and Related Techniques, Doll, T., Ed. Springer US: Boston, MA, 2003; pp 123-159.

47. Chang, S. C., Oxygen chemisorption on tin oxide: Correlation between electrical conductivity and EPR measurements. Journal of Vacuum Science and Technology 1980, 17, 366-369.

48. Yamazoe, N.; Shimanoe, K., Theory of Power Laws for Semiconductor Gas Sensors. Sens. Actuators B 2008, 128, 566-573.

49. European-Environment-Agency Air quality standards. https:/www.eea.europa.eu/themes/air/air-quality-standards (accessed 21.01.2018).

50. Glorennec, P.; Bonvallot, N.; Mandin, C.; Goupil, G.; Pernelet-Joly, V.; Millet, M.; Filleul, L.; Le Moullec, Y.; Alary, R., Is a quantitative risk assessment of air quality in underground parking garages possible? Indoor Air 2008, 18, 283-292.

51. Levy, J. I.; Lee, K.; Yanagisawa, Y.; Hutchinson, P.; Spengler, J. D., Determinants of nitrogen dioxide concentrations in indoor ice skating rinks. American Journal of Public Health 1998, 88, 1781-1786.

52. Hernández-Ramírez, F.; Tarancón, A.; Casals, O.; Arbiol, J.; Romano-Rodríguez, A.; Morante, J. R., High Response and Stability in $\mathrm{CO}$ and Humidity Measures Using a Single SnO2 Nanowire. Sens. Actuators B 2007, 121, 3-17.

53. Kim, Y. S.; Ha, S.-C.; Kim, K.; Yang, H.; Choi, S.-Y.; Kim, Y. T.; Park, J. T.; Lee, C. H.; Choi, J.; Paek, J.; Lee, K., RoomTemperature Semiconductor Gas Sensor Based on nonstoichiometric Tungsten Oxide Nanorod Film. Appl. Phys. Lett. 2005, 86, 213105.

54. Penza, M.; Tagliente, M. A.; Mirenghi, L.; Gerardi, C.; Martucci, C.; Cassano, G., Tungsten Trioxide (WO3) Sputtered Thin Films for a NOx Gas Sensor. Sens. Actuators B 1998, 50, 9-18.

55. Jolliffe, I. T., Principal Component Analysis and Factor Analysis. In Principal Component Analysis, Springer New York: New York, NY, 1986; pp 115-128.

56. Jolliffe, I. T., Principal Component Analysis. 2nd ed.; Springer New York, 2002. 